\title{
Signal Transduction
}

National Cancer Institute

\section{Source}

National Cancer Institute. Signal Transduction. NCI Thesaurus. Code C17133.

A cellular process that involves a chemical or mechanical stimulus and results in a specific cellular response. This process is a central feature of a broad variety of physiologic responses. 